\title{
Upper Limit of Normal
}

National Cancer Institute

\section{Source}

National Cancer Institute. Upper Limit of Normal. NCI Thesaurus. Code C25706.

The top value of the range of statistical characteristics that are supposed to represent accepted standard, non-pathological pattern for particular laboratory result or other quantitative parameter. 